\title{
INTEGRAL OPERATORS ON THE SECTION SPACE OF A BANACH BUNDLE
}

\author{
J.W. KITCHEN \\ Department of Mathematics \\ Duke University \\ Durham, NC 27706 USA \\ and \\ D.A. ROBBINS \\ Department of Mathematics \\ Trinity College \\ Hartford, CT 06106 USA
}

(Received December 5, 1991 and in revised form August 15, 1992)

\begin{abstract}
Let $\pi: \mathrm{E} \rightarrow \mathrm{X}$ and $\rho: \mathrm{F} \rightarrow \mathrm{X}$ be bundles of Banacn spaces, where $\mathrm{X}$ is a compact Hausdorff space, and let $\mathrm{V}$ be a Banach space. Let $\Gamma(\pi)$ denote the space of sections of the bundle $\pi$. We obtain two representations of integral operators $T: \Gamma(\pi) \rightarrow V$ in terms of measures. The first generalizes a recent result of $\mathrm{P}$. Saab, the second generalizes a theorem of Grothendieck. We also study integral operators $\mathrm{T}: \Gamma(\boldsymbol{x}) \rightarrow \Gamma(\rho)$ which are $\mathrm{C}(\mathrm{X})$-linear.
\end{abstract}

KEYWORDS AND PHRASES. Banach bundle, section space, integral operator, canonical bundle of a Banach module.

1991 AMS SUBJECT CLASSIFICATION CODES. Primary 46H25; Secondary 46M05, 46E40, 46B28.

\section{INTRODUCTION.}

A bounded operator $\mathrm{T}: \mathrm{V} \rightarrow \mathrm{W}$ between Banach spaces is called an integral operator (in the sense of Grothendieck) if there is a bounded linear functional $\phi$ on the inductive tensor product $\mathrm{V} \hat{\hat{\otimes}} \mathrm{W}^{*}$ such that $\phi\left(x \otimes y^{*}\right)=y^{*}(\mathrm{~T}(x))$ for all $x \in \mathrm{V}$ and $y^{*} \in \mathrm{W}^{*}$. The integral operator $\mathrm{T}: \mathrm{V} \rightarrow$ $\mathrm{W}$ carries a norm $\|\mathrm{T}\|$ int $\leq\|\mathrm{T}\|$ which is the norm of the functional $\tau_{\mathrm{T}} \in\left(\mathrm{V} \otimes \mathrm{W}^{*}\right)^{*}$, where $\tau_{\mathrm{T}}\left(x \otimes y^{*}\right)=y^{*}(\mathrm{~T}(x))$. In this paper, we study integral operators $\mathrm{T}: \Gamma(\pi) \rightarrow \mathrm{W}$, where $\pi: \mathrm{E} \rightarrow \mathrm{X}$ is a Banach bundle and the base space $X$ is compact Hausdorff. In Section 3, we obtain two representations of such operators by means of measures. The first of these is described in Theorem 3, which generalizes a result of $P$. Saab [1] for integral operators on $C(X, V)$, the space of continuous functions from $X$ to a Banach space $V$. (Of course, $C(X, V)=\Gamma(\pi)$, when $\pi$ is the trivial bundle whose fiber space is $\mathrm{X} \times \mathrm{V}$ with its product topology.) The second representation occurs as a corollary to Theorem 4, which itself generalizes a theorem of Grothendieck [2]. Finally, in Section 4, we study integral operators $T: \Gamma(x) \rightarrow \Gamma(\rho)$ which are $\mathrm{C}(\mathrm{X})$-linear, where $\pi$ and $\rho$ are Banach bundles with base space $X$.

In this paper, the base space $\mathrm{X}$ of a Banach bundle $x: \mathrm{E} \rightarrow \mathrm{X}$ is always assumed to be compact and Hausdorff. The reader is referred to Kitchen and Robbins [3] for details about the canonical bundle $\pi: \mathrm{E} \rightarrow \mathrm{X}$ of a Banach module $\mathrm{M}$ over $\mathrm{C}(\mathrm{X})$ and the Gelfand representation of $M$ as a space of sections in $\Gamma(\pi)$.

Our two representations of integral operators on $\Gamma(\pi)$ involve a compact Hausdorff space 8 , which we shall call the carrier space of the Banach bundle $\pi: \mathrm{E} \rightarrow \mathrm{X}$. The space 8 was introduced by A. Seda in [4], and is described in Section 2.

2. PRELIMINARIES.

Throughout the paper, we shall use $\mathrm{B}(\mathrm{V})$ to denote the closed unit ball of the Banach space 
$\mathrm{V}$; that is, $\mathrm{B}(\mathrm{V})=\{x \in \mathrm{V}:\|x\| \leq 1\}$. In particular, we shall use this notation in describing the carrier space $\varepsilon$ of a Banach bundle $\pi: \mathrm{E} \rightarrow \mathrm{X}$. If $p \in \mathrm{X}$, then we denote by $\mathrm{E}_{p}$ the fiber above $p$; that is, $\mathrm{E}_{p}=\pi^{-1}(\{p\})$. As a point set the space $8 \mathrm{can}$ be described as the disjoint union of the unit balls of the dual space of the fibers. Thus

$$
\begin{aligned}
8 & =\bigcup_{p \in \mathrm{X}} \mathrm{B}\left(\left(\mathrm{E}_{p}\right)^{*}\right) \times\{p\} \\
& =\left\{(f, p): p \in \mathrm{X}, f \in\left(\mathrm{E}_{p}\right)^{*},\|f\| \leq 1\right\} .
\end{aligned}
$$

There are two important maps associated with $8:$ the obvious coordinate projection $\pi: 8 \rightarrow \mathrm{X}$, defined by $\pi((f, p))=p$, and a map $\Phi: 8 \rightarrow \mathrm{B}\left(\Gamma(\pi)^{*}\right)$. The map $\Phi$ assigns to the point $(f, p)$ in 8 the function $\mathrm{F}_{f, p}: \Gamma(\boldsymbol{x}) \rightarrow \mathrm{C}$ defined by

$$
\mathrm{F}_{f, p}(\sigma)=f(\sigma(p))
$$

for all $\sigma \in \Gamma(\pi)$. It is easily verified that $F_{f, p}$ is a bounded linear functional on $\Gamma(\pi)$ whose norm is the same as the norm of $f$. As a result, $\Phi$ maps 8 into $\mathrm{B}\left(\Gamma(\pi)^{*}\right)$ as we already noted. We topologize 8 by giving it the weakest topology which makes the maps $\pi: 8 \rightarrow \mathrm{X}$ and $\Phi: \mathcal{8} \rightarrow$ $\left.\mathrm{B}\left(\Gamma(\pi)^{*}\right)\right)$ continuous, where $\mathrm{B}\left(\Gamma(\pi)^{*}\right)$ is given its compact weak-* topology. It follows that a net $\left\{\left(f_{\alpha}, p_{\alpha}\right)\right\}$ in 8 converges to $(f, p)$ if and only if

$$
\lim p_{\alpha}=p \text { and } \lim f_{\alpha}\left(\sigma\left(p_{\alpha}\right)\right)=f(\sigma(p)) \text { for all } \sigma \text { in } \Gamma(\pi) .
$$

When 8 is topologized in this way, the projection $\pi: 8 \rightarrow \mathrm{X}$ is not only continuous, but open, and for each $p \in \mathrm{X}$, the topology which $\mathrm{B}\left(\left(\mathrm{E}_{p}\right)^{*}\right)$ inherits from 8 is its compact weak-* topology as a subset of $\left(E_{p}\right)^{*}$. (See Seda [4] or Kitchen and Robbins [5] for a further description of the topology on 8.) The space $\mathrm{C}(8)$ can be viewed as a $\mathrm{C}(\mathrm{X})$-module in a natural way. Given $a \in \mathrm{C}(\mathrm{X})$ and $g \in \mathrm{C}(\mathcal{8})$, we define $a g$ to be the pointwise product $(a \circ \pi) g$; thus, for all $(f, p) \in \mathcal{8}$

$$
(a g)((f, p))=a(p) g((f, p)) .
$$

Finally, there is an important embedding $I: \Gamma(\pi) \rightarrow \mathrm{C}(8)$. Given $\sigma \in \Gamma(\pi), \quad \mathrm{I}(\sigma)$ is the function $\tilde{\sigma}: B \rightarrow C$ defined by

$$
\widetilde{\sigma}((f, p))=\mathrm{F}_{f, p}(\sigma)=f(\sigma(p)) .
$$

From our definition of the topology on 8 , it is clear that $\widetilde{\sigma}$ is continuous. The most important facts concerning 8 are contained in

PROPOSITION 1. Let $\pi: \mathrm{E} \rightarrow \mathrm{X}$ be a Banach bundle. The carrier space $\varepsilon$ is compact Hausdorff and the map $I: \Gamma(\pi) \rightarrow C(8)$ is a $C(X)$-linear isometry.

PROOF. The compactness of 8 follows rather easily from the compactness of $X$ and of $\mathrm{B}\left(\Gamma(\pi)^{*}\right)$ ). (Seda [4] proves that 8 is locally compact under the assumption that $\mathrm{X}$ is locally compact.) Let $\left\{\left(f_{\alpha}, p_{\alpha}\right)\right\}$ be a net in 8 . Since $\mathrm{X}$ is compact, there exists a subnet $\left\{p_{\beta}\right\}$ of $\left\{p_{\alpha}\right\}$ such that $p_{\beta} \rightarrow p$ in $X$. Now, consider the subnet $\left\{\left(f_{\beta}, p_{\beta}\right)\right\}$ of $\left\{\left(f_{\alpha}, p_{\alpha}\right)\right\}$. As before, define $\tilde{f}_{\beta}$ $\in \Gamma(\pi)^{*}$ by $\tilde{f}_{\beta}(\sigma)=f_{\beta}\left(\sigma\left(p_{\beta}\right)\right)=F_{f_{\beta}, p_{\beta}}(\sigma)$. Then $\tilde{f}_{\beta}$ is a net in $\mathrm{B}\left(\Gamma(\pi)^{*}\right)$ which converges weak-* to some $g \in \mathrm{B}\left(\Gamma(\pi)^{*}\right)$. But note that for $\sigma \in \Gamma(\pi)$ and $a \in \mathrm{C}(\mathrm{X})$, we have

$$
\begin{aligned}
g(a \sigma) & =\lim \tilde{f}_{\beta}(a \sigma)=\lim a\left(p_{\beta}\right) \tilde{f}_{\beta}(\sigma) \\
& =a(p) g(\sigma) ;
\end{aligned}
$$

i.e. $(a g)(\sigma)=a(p) g(\sigma)$. It follows from e.g. Gierz [6] that $g=\tilde{f}$ for some $f \in \mathrm{B}\left(\left(\mathrm{E}_{p}\right)^{*}\right)$, and it is clear that $\left(f_{\beta}, p_{\beta}\right) \rightarrow(f, p)$ in the topology on 8 .

The first part of the statement is obvious from the definition of the module operation on $\mathrm{C}(8)$.

The next result is known. We include its statement here for easy reference.

PROPOSITION 2. Suppose that $\Theta: \mathrm{Z} \rightarrow \mathrm{V}$ and $\Psi: \mathrm{Y} \rightarrow \mathrm{W}$ are bounded linear maps between Banach spaces. Then the tensor product map $\Theta \otimes \Psi: \mathrm{Z} \otimes \mathrm{Y} \rightarrow \mathrm{V} \otimes \mathrm{W}$ extends uniquely to a bounded linear map 
such that $\|\Theta \otimes \Psi\| \leq\|\Theta\|\|\Psi\|$. If $\Theta$ and $\Psi$ are isometries, then $\Theta \otimes \Psi$ is also an isometry.

PROOF. The proof of the first assertion can be found in Diestel and Uhl [7, p. 228].

Suppose now that $\Theta$ and $\Psi$ are isometries. Then $\Theta \otimes \Psi=\left(\mathrm{id}_{Z} \otimes \Psi\right) \circ\left(\Theta \otimes \mathrm{id}_{\mathrm{W}}\right)$, where $\mathrm{id}_{\mathrm{Z}} \otimes \Psi: \mathrm{Z} \hat{\hat{\otimes}} \mathrm{Y} \rightarrow \mathrm{Z} \hat{\hat{\otimes}} \mathrm{W}$ and $\Theta \otimes \mathrm{id}_{\mathrm{W}}: \mathrm{Z} \hat{\hat{\otimes}} \mathrm{W} \rightarrow \mathrm{V} \hat{\hat{\otimes}} \mathrm{W}$, and $\mathrm{id}_{\mathrm{Z}}$ and $\mathrm{id} W$ are the identity maps on $Z$ and $W$, respectively. By Diestel and Uhl [7, p. 225], both $\mathrm{id}_{Z} \otimes \Psi$ and $\Theta \otimes i d_{W}$ are isometries, and hence so is $\Theta \otimes \Psi$. $ם 00$

\section{REPRESENTATION OF INTEGRAL OPERATORS BY MEASURES.}

The first result of this section is a generalization of a result of P. Saab to operators on the section space of a Banach bundle.

THEOREM 3. Let $\pi: \mathrm{E} \rightarrow \mathrm{X}$ be a Banach bundle. Let $\mathrm{V}$ be a Banach space, and let $\mathrm{T}$ : $\Gamma(\pi) \rightarrow V$ be a bounded linear map. Then $T$ is an integral operator iff there exists a regular $V^{* *}$. valued Borel measure $\mu$ on $\delta$, the carrier space for $\pi$, such that

$$
(\mathrm{J} \circ \mathrm{T})(\sigma)=\int_{\mathbb{B}} f(\sigma(p)) \mathrm{d} \mu(f, p)
$$

for all $\sigma \in \Gamma(\pi)$, where $\mathrm{J}: \mathrm{V} \rightarrow \mathrm{V}^{* *}$ is the natural embedding.

PROOF. Suppose that there is a measure $\mu \in M\left(\varepsilon, V^{* *}\right)$, the space of regular, $V^{* *}$-valued Borel measures on 8, for which (*) holds. We will show that $\mathrm{T}$ is an integral operator.

Since the space $\mathrm{M}\left(\varepsilon, \mathrm{V}^{* *}\right)$ of all regular Borel $\mathrm{V}^{* *}$-valued measures on 8 which are of bounded variation is isometrically isomorphic to the space of integral operators from $C(8)$ to $\mathrm{V}^{* *}$ ', it follows that if $\mu \in \mathrm{M}\left(\varepsilon, \mathrm{V}^{* *}\right)$ is such that $(*)$ holds, then the operator $\widehat{\mathrm{T}}: \mathrm{C}(8) \rightarrow \mathrm{V}^{* *}$ defined by

$$
\widehat{\mathrm{T}}(g)=\int_{8} g \mathrm{~d} \mu, \text { for } g \in \mathrm{C}(\boldsymbol{\varepsilon}),
$$

is an integral operator. Thus, if $I: \Gamma(\pi) \rightarrow C(8)$ denotes the natural embedding described earlier. that is

$$
\{\mathrm{I}(\sigma)\}(f, p)=f(\sigma(p))
$$

for all $(f, p) \in \mathcal{8}$, then $(*)$ states that $(\mathrm{J} \circ \mathrm{T})(\sigma)=(\widehat{\mathrm{T}} \circ \mathrm{I})(\sigma)$ for all $\sigma \in \Gamma(\pi)$, that is, the diagram

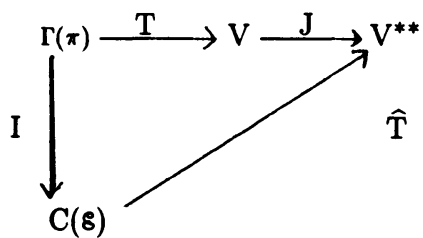

commutes.

Since $\widehat{\mathrm{T}} \circ \mathrm{I}$ is integral, it follows that $\mathrm{J} \circ \mathrm{T}$ is integral, and hence that $\mathrm{T}$ is integral, by Diestel and $\mathrm{Uhl}[7$, p. 233].

Now, suppose conversely that $\mathrm{T}$ is an integral operator. Then there exists a bounded linear functional $\Theta$ on $\Gamma(\pi) \hat{\hat{\theta}} V^{*}$ such that

$$
\Theta\left(\sigma \otimes v^{*}\right)=v^{*}(\mathrm{~T}(\sigma))
$$

for all $\sigma \in \Gamma(x)$ and $v^{*} \in V^{*}$. Since $I: \Gamma(x) \rightarrow C(8)$ and id $: V^{*} \rightarrow V^{*}$ are isometries, the map $I \otimes$ id $: \Gamma(\pi) \hat{\hat{\otimes}} V^{*} \rightarrow C(8) \hat{\hat{\otimes}} V^{*}$ is an isometry, whose range, W, is a closed subspace on which the linear functional $\Theta \circ(I \otimes i d)^{-1}$ is bounded. By the Hahn-Banach theorem, this functional can be extended without increase in norm to a bounded linear functional $\widetilde{\Theta}$ on $C(\varepsilon) \hat{\hat{\otimes}} \mathrm{V}^{*}$. Then for all $\sigma \in \Gamma(\pi)$ and $v^{*} \in \mathrm{V}^{*}$

$$
\widetilde{\Theta}\left(\mathrm{I}(\sigma) \otimes v^{*}\right)=\widetilde{\Theta}\left((\mathrm{I} \otimes \mathrm{id})\left(\sigma \otimes v^{*}\right)\right)=\Theta\left(\sigma \otimes v^{*}\right) .
$$

Since $\widetilde{\theta}$ belongs to the dual space of $\mathrm{C}(8) \hat{\hat{\otimes}} \mathrm{V}^{*} \simeq \mathrm{C}\left(8, \mathrm{~V}^{*}\right)$, there is a unique measure $\mu \in \mathrm{M}(8$, $\left.\mathrm{V}^{* *}\right)$ such that for all $g \in \mathrm{C}(8)$ and $v^{*} \in \mathrm{V}^{*}$,

$$
\widetilde{\Theta}\left(g \otimes v^{*}\right)=\int_{\delta} g \mathrm{~d}\left\langle v^{*}, \mu\right\rangle,
$$


where $\left\langle v^{*}, \mu\right)$ is the $\mathrm{C}$-valued Borel measure such that

$$
\left\langle v^{*}, \mu\right\rangle(\mathrm{B})=\{\mu(\mathrm{B})\}\left(v^{*}\right)=\mathrm{J}^{\prime \prime}\left(v^{*}\right)\{\mu(\mathrm{B})\}=\left(\mathrm{J}^{\prime \prime}\left(v^{*}\right) \circ \mu\right)(\mathrm{B})
$$

for every Borel subset of 8 (where $\mathrm{J}^{\prime \prime}$ is the natural embedding of $\mathrm{V}^{*}$ into $\mathrm{V}^{* * *}$ ). We must now show that

$$
(\mathrm{J} \circ \mathrm{T})(\sigma)=\int_{\mathcal{E}} f(\sigma(p)) \mathrm{d} \mu(f, p)
$$

for all $\sigma \in \Gamma(\pi)$. Now, for all $v^{*} \in \mathrm{V}^{*}$,

$$
\begin{aligned}
\{(\mathrm{J} \circ \mathrm{T})(\sigma)\}\left(v^{*}\right) & =v^{*}(T(\sigma))=\Theta\left(\sigma \otimes v^{*}\right)=\widetilde{\Theta}\left(\mathrm{I}(\sigma) \otimes v^{*}\right) \\
& =\int_{\delta} \mathrm{I}(\sigma) \mathrm{d}\left(\mathrm{J}^{\prime \prime}\left(v^{*}\right) \circ \mu\right)=\mathrm{J}^{\prime \prime}\left(v^{*}\right)\left(\int_{\mathcal{B}} \mathrm{I}(\sigma) \mathrm{d} \mu\right)=\left(\int_{\mathcal{\delta}} \mathrm{I}(\sigma) \mathrm{d} \mu\right)\left(v^{*}\right) .
\end{aligned}
$$

Thus,

$$
(\mathrm{J} \circ \mathrm{T})(\sigma)=\int_{\delta} \mathrm{I}(\sigma) \mathrm{d} \mu=\int_{\delta} f(\sigma(p)) \mathrm{d} \mu(f, p) . \quad \square 00
$$

In the special case when $\Gamma(\pi)=\mathrm{C}(\mathrm{X}, \mathrm{V})$ for a Banach space $\mathrm{V}, 8$ is the product space $\mathrm{X} \times \mathrm{B}\left(\mathrm{V}^{*}\right)$, and we recover Saab's result. The effect is to reduce the space on which the measures lives to be as small as possible, and the result may find its relevance in the fact (see e.g. Behrends [8]) that every Banach space is isometrically isomorphic to a space of sections over its center.

Our next result generalizes a result of Grothendieck [2] to Banach bundles; our proof is similar to that of Diestel and Uhl [7] for the special case of continuous bilinear maps from the inductive tensor $\mathrm{V} \hat{\hat{\theta}} \mathrm{W}$ of Banach spaces $\mathrm{V}$ and $\mathrm{W}$ to $\mathrm{C}$.

THEOREM 4. Let $\pi: \mathrm{E} \rightarrow \mathrm{X}$ be a Banach bundle, and let $\varepsilon$ be its compact carrier space. Let $\mathrm{V}$ be a Banach space, and give $\mathrm{B}\left(\mathrm{V}^{*}\right)$ its compact weak-* topology. A bilinear functional $\psi$ on $\Gamma(\pi) \times \mathrm{V}$ defines a member of $(\Gamma(\pi) \hat{\hat{\theta}} \mathrm{V})^{*}$ iff there exists a regular Borel measure $\mu$ on $8 \times \mathrm{B}\left(\mathrm{V}^{*}\right)$ such that

$$
\psi(\sigma, y)=\int_{8 \times \mathrm{B}\left(\mathrm{V}^{*}\right)} f(\sigma(p)) y^{*}(y) \mathrm{d} \mu\left((f, p), y^{*}\right)
$$

for all $\sigma \in \Gamma(x)$ and $y \in V$. In this case the norm of $\psi$ as a member of $(\Gamma(x) \hat{\hat{\otimes}} V)^{*}$ is the variation $|\mu|\left(8 \times \mathrm{B}\left(\mathrm{V}^{*}\right)\right)$ of $\mu$.

PROOF. Suppose that $\psi$ extends to an element (which we will also denote by $\psi$ ) in $(\Gamma(x) \hat{\hat{\theta}} \mathrm{V})^{*}$. We let $\mathrm{I}: \Gamma(\pi) \rightarrow \mathrm{C}(\boldsymbol{8})$ and $\mathrm{i}: \mathrm{V} \rightarrow \mathrm{C}\left(\mathrm{B}\left(\mathrm{V}^{*}\right)\right)$ be the natural isometries. Thus,

$$
\{\mathrm{I}(\sigma)\}(f, p)=f(\sigma(p)) \text { and }\left\{\mathrm{i}\left(y^{*}\right)\right\}(y)=y^{*}(y)
$$

for all $\sigma \in \Gamma(x), y \in \mathrm{V},(f, p) \in \mathcal{8}$ and $y^{*} \in \mathrm{B}\left(\mathrm{V}^{*}\right)$. Now, the tensor product map $\mathrm{I} \otimes \mathrm{i}: \Gamma(\boldsymbol{x}) \hat{\hat{\otimes}} V \rightarrow \mathrm{C}(\boldsymbol{8}) \hat{\hat{\otimes}} \mathrm{C}\left(\mathrm{B}\left(\mathrm{V}^{*}\right)\right)$ is an isometry such that

$$
(\mathrm{I} \otimes \mathrm{i})(\sigma \otimes y)=\mathrm{I}(\sigma) \otimes \mathrm{i}(y)
$$

for all $\sigma \in \Gamma(\pi)$ and $y \in \mathrm{V}^{*}$. If $\Theta: \mathrm{C}(8) \hat{\hat{\otimes}} \mathrm{C}\left(\mathrm{B}\left(\mathrm{V}^{*}\right)\right) \rightarrow \mathrm{C}\left(8 \times \mathrm{B}\left(\mathrm{V}^{*}\right)\right)$ is the natural isometric isomorphism, then the map $\mathrm{R}=\Theta \circ(\mathrm{I} \otimes \mathrm{i}): \Gamma(\pi) \hat{\hat{\otimes}} \mathrm{V} \rightarrow \mathrm{C}\left(8 \times \mathrm{B}\left(\mathrm{V}^{*}\right)\right)$ is an isometry which is characterized by the equation

$$
\{\mathrm{R}(\sigma \otimes y)\}\left((f, p), y^{*}\right)=f(\sigma(p)) y^{*}(y)
$$

for all $\sigma \in \Gamma(\pi), y \in \mathrm{V},(f, p) \in \mathcal{8}$, and $y^{*} \in \mathrm{B}\left(\mathrm{V}^{*}\right)$. If we let $\mathrm{W}$ be the range of $\mathrm{R}$, then $\mathrm{W}$ is a closed subspace of $C\left(8 \times B\left(V^{*}\right)\right)$ and $R: \Gamma(\pi) \hat{\hat{\theta}} V \rightarrow W$ is a bijective linear isometry. Thus, the bounded linear functional $\psi \circ \mathrm{R}^{-1}$ on $\mathrm{W}$ can be extended without increase in norm to a bounded linear functional $\phi$ on $\mathrm{C}\left(8 \times \mathrm{B}\left(\mathrm{V}^{*}\right)\right)$. Moreover, $\|\phi\|=\left\|\psi \circ \mathrm{R}^{-1}\right\|=\|\psi\|$. By the Riesz representation theorem, there exists a Borel measure $\mu$ on $8 \times \mathrm{B}\left(\mathrm{V}^{*}\right)$ such that $\phi(g)=\int g \mathrm{~d} \mu$, for all $g \in \mathrm{C}$ $\left(8 \times \mathrm{B}\left(\mathrm{V}^{*}\right)\right)$. Thus, for all $\sigma \in \Gamma(\pi)$ and $y \in \mathrm{V}$, we have

$$
\begin{aligned}
\psi(\sigma, y) & =\psi(\sigma \otimes y)=\left(\psi \circ \mathrm{R}^{-1}\right)(\mathrm{R}(\sigma \otimes y)=\phi(\mathrm{R}(\sigma \otimes y)) \\
& =\int_{8 \times \mathrm{B}\left(\mathrm{V}^{*}\right)} \mathrm{R}(\sigma \otimes y) \mathrm{d} \mu=\int_{8 \times \mathrm{B}\left(\mathrm{V}^{*}\right)} f(\sigma(p)) y^{*}(y) \mathrm{d} \mu\left((f, p), y^{*}\right)
\end{aligned}
$$


Moreover, the norm of $\psi$ as an element of $(\Gamma(\pi) \hat{\hat{\otimes}} \mathrm{V})^{*}$ is equal to $\|\phi\|=\|\mu\|=$ the variation of $\mu$ on $8 \times B\left(V^{*}\right)$.

Suppose conversely that there is a Borel measure $\mu$ on $8 \times B\left(V^{*}\right)$ such that

$$
\psi(\sigma, y)=\int_{8 \times \mathrm{B}\left(\mathrm{V}^{*}\right)} \mathrm{R}(\sigma \otimes y) \mathrm{d} \mu
$$

for all $\sigma \in \Gamma(\pi)$ and $y \in V$. If we let $\phi$ be the bounded linear functional on $C\left(8 \times B\left(V^{*}\right)\right)$ which corresponds to $\mu$, then according to the above equation,

$$
\psi(\sigma, y)=\phi(\mathrm{J}(\sigma \otimes y))
$$

for all $\sigma \in \Gamma(\pi)$ and $y \in \mathrm{V}$, which is to say that $\phi \circ \mathrm{R}$ is a bounded linear functional on $\Gamma(\pi) \hat{\hat{\theta}} \mathrm{V}$ which extends the bilinear functional $\psi$. $\mathrm{QDO}$

COROLlARY 5. Let $\pi: \mathrm{E} \rightarrow \mathrm{X}$ and $\mathrm{V}$ be as above. If $\mathrm{T}: \Gamma(\boldsymbol{x}) \rightarrow \mathrm{V}$ is an integral operator. then there exists a Borel measure $\mu$ on $\varepsilon \times B\left(V^{* *}\right)$ such that

for all $\sigma \in \Gamma(\pi)$ and $y^{*} \in V^{*}$.

$$
y^{*}(\mathrm{~T}(\sigma))=\int_{8 \times \mathrm{B}\left(\mathrm{V}^{* *}\right)} f(\sigma(p)) \eta\left(y^{*}\right) \mathrm{d} \mu((f, p), \eta)
$$

PROOF. Because $T: \Gamma(x) \rightarrow V$ is an integral operator, the continuous bilinear functional $\psi$ on $\Gamma(\pi) \times \mathrm{V}^{*}$ defined by $\psi\left(\sigma, y^{*}\right)=y^{*}(T(\sigma))$ determines a bounded linear functional on $\Gamma(\pi) \hat{\hat{\otimes}} \mathrm{V}^{*}$, so the theorem guarantees the existence of a Borel measure $\mu$ on $8 \times \mathrm{B}\left(\mathrm{V}^{* *}\right)$ with the required properties. $\mathrm{QDO}$

\section{THE SPACE OF INTEGRAL C(X)-MODULE OPERATORS AS A C(X)-MODULE.}

We turn now to the study of the space of integral operators $T$ between sections spaces of Banach bundles which also have the property that $\mathrm{T}$ is a $\mathrm{C}(\mathrm{X})$-module homomorphism. That is if $\pi: \mathrm{E} \rightarrow \mathrm{X}$ and $\rho: \mathrm{F} \rightarrow \mathrm{X}$ are bundles, then $\mathrm{T}: \Gamma(\pi) \rightarrow \Gamma(\rho)$ will satisfy the equation $(a \mathrm{~T})(\sigma)=$ $a \cdot \mathrm{T}(\sigma)=\mathrm{T}(a \sigma)$ for each $a \in \mathrm{C}(\mathrm{X})$ and $\sigma \in \Gamma(\pi)$.

LEMMA 6. Let $\pi: \mathrm{E} \rightarrow \mathrm{X}$ be a Banach bundle, and let $\mathrm{V}$ be a Banach space. The space

$$
\mathrm{M}=\Gamma(\pi) \hat{\hat{\otimes}} \mathrm{V}
$$

can be made into a $\mathrm{C}(\mathrm{X})$-module which is $\mathrm{C}(\mathrm{X})$-locally convex. If $\xi: \mathrm{G} \rightarrow \mathrm{X}$ is the canonica! bundle for $M$, then for each $p \in X$, the stalk $G_{p}=\xi^{-1}(p)$ can be identified with $E_{p} \hat{\hat{\otimes}} Y$ Moreover, if $\mathrm{ev}_{p}: \Gamma(\boldsymbol{x}) \rightarrow \mathrm{E}_{p}$ is evaluation at $p$ and id $: \mathrm{V} \rightarrow \mathrm{V}$ is the identity map, then $\mathrm{ev}_{p} \otimes 1 \mathrm{~d}$. $\Gamma(\pi) \hat{\hat{\otimes}} \mathrm{V} \rightarrow \mathrm{E}_{p} \hat{\hat{\otimes}} \mathrm{V}$ is a quotient map whose kernel is $\mathrm{I}_{p} \mathrm{M}=\{a m: m \in \mathrm{M}, a \in \mathrm{C}(\mathrm{X})$, and $a(p)=0\}$.

PROOF. Given $a \in \mathrm{C}(\mathrm{X})$, we let $\mu_{a}: \Gamma(\pi) \rightarrow \Gamma(\pi)$ be pointwise multiplication by $a$. The tensor product of $\mu_{a}$ with id $: \mathrm{V} \rightarrow \mathrm{V}$ then yields a bounded linear operator

$$
\mu_{a} \otimes \mathrm{id}: \Gamma(\pi) \hat{\hat{\otimes}} \mathrm{V} \rightarrow \Gamma(\pi) \hat{\hat{\otimes}} \mathrm{V}
$$

on $\mathrm{M}$, and $\left\|\mu_{a} \otimes \mathrm{id}\right\| \leq\left\|\mu_{a}\right\|$ id $\|=\| a \|$. We make $\mathrm{M}$ into a $\mathrm{C}(\mathrm{X})$-module by defining

$$
a m=\left(\mu_{a} \otimes \mathrm{id}\right)(m)
$$

for all $a \in \mathrm{C}(\mathrm{X})$ and $m \in \mathrm{M}$. Then

$$
\|a m\| \leq\left\|\mu_{a} \otimes \mathrm{id}\right\|\|m\| \leq\|a\|\|m\|
$$

and we have

$$
a(\sigma \otimes f)=\left(\mu_{a} \otimes \mathrm{id}\right)(\sigma \otimes f)=\mu_{a}(\sigma) \otimes \mathrm{id}(f)=(a \sigma) \otimes f
$$

for all $a \in \mathrm{C}(\mathrm{X}), \sigma \in \mathrm{\Gamma}(\pi)$ and $f \in \mathrm{V}$. It is then straightforward to complete the verification that in this way $\mathrm{M}$ becomes a $\mathrm{C}(\mathrm{X})$-module.

We next apply Theorem 4.2 of Kitchen and Robbins [9]. We can view $\mathrm{V}$ as the section space $\Gamma\left(1_{\mathrm{V}}\right)$, where $1_{\mathrm{V}}$ denotes the Banach bundle whose base space is the singleton set $\{1\}$ and whose one stalk is V. Then, according to the theorem cited, $M=\Gamma(\pi) \hat{\hat{\otimes}} V=\Gamma(\pi) \hat{\hat{\otimes}} \Gamma(1, V)$ is isometrically isomorphic to $\Gamma\left(\pi \hat{\hat{\otimes}} 1_{V}\right)$. The bundle $\pi \hat{\hat{\otimes}} 1 \mathrm{~V}$ has $\mathrm{X} \times\{1\}$ as its base space, and, for

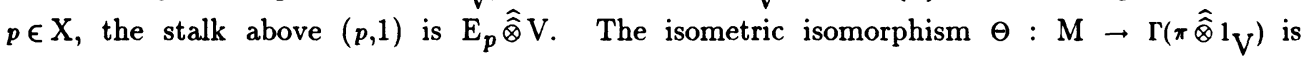


characterized by the equation $\Theta(\sigma \otimes y)=\sigma \odot y$ for all $\sigma \in \Gamma(x)$ and $y \in \mathrm{V}$, where

$$
(\sigma \odot y)(p, 1)=\sigma(p) \otimes y(1)=\sigma(p) \otimes y .
$$

(In other words, we identify an element $y \in \mathrm{V}$ with the section in $\Gamma\left(1_{\mathrm{V}}\right)$ whose (one and only) value is $y$.) Now, $\Gamma(\pi \hat{\hat{\otimes}} 1 V)$ is a Banach module over $C(X \times\{1\})$. By identifying $X \times\{1\}$ with $X$ in the obvious way, we can view $\Gamma(\pi \hat{\hat{\otimes}} 1 \mathrm{~V})$ as a $\mathrm{C}(\mathrm{X})$-module.

We will now show that our isometric isomorphism $\Theta: M \rightarrow \Gamma\left(\pi \hat{\hat{\otimes}} 1 V^{\prime}\right)$ is $C(X)$-linear. It suffices to show that

$$
\Theta(a(\sigma \otimes f))=a \Theta(\sigma \otimes f)
$$

holds for all $a \in \mathrm{C}(\mathrm{X}), \sigma \in \Gamma(\pi)$, and $f \in \mathrm{V}$. But

$$
\Theta(a(\sigma \otimes f))=(a \sigma) \otimes f=\{a(p) \sigma(p)\} \otimes f=a(p)\{\sigma(p) \otimes f\},
$$

so that $a \sigma \odot f=a(\sigma \odot f)=a \Theta(\sigma \otimes f)$. Thus,

$$
\Theta(a(\sigma \otimes f))=a \Theta(\sigma \otimes f) .
$$

Hence, $M$ and $\Gamma(\pi \hat{\hat{\otimes}} 1 V)$ are isomorphic as $C(X)$-modules. Since $\Gamma(\pi \hat{\hat{\otimes}} 1 V)$ is $C(X)$-locally convex (because of identification of $\mathrm{C}(\mathrm{X})$ with $\mathrm{C}(\mathrm{X} \times\{1\})), \mathrm{M}$ is $\mathrm{C}(\mathrm{X})$-locally convex. It follows that the canonical bundle $\xi: G \rightarrow X$ is bundle isomorphic to $\pi \hat{\hat{\otimes}} 1 \mathrm{~V}$. Thus, for $p \in X$, the stalk $G_{p}$ can be identified with $E_{p} \hat{\hat{\theta}} \mathrm{V}$, in which case the Gelfand representation of an element $m \in M$ is given by

Thus, if $\sigma \in \Gamma(\pi)$ and $f \in \mathrm{V}$,

$$
\hat{m}(p)=\{\Theta(m)\}(p) .
$$

$$
(\sigma \otimes f) \hat{(p)}=\{\Theta(\sigma \otimes f)\}(p)=(\sigma \odot f)(p)=\sigma(p) \otimes f=\left(\operatorname{ev}_{p} \otimes \mathrm{id}\right)(\sigma \otimes f),
$$

where $\operatorname{ev}_{p}: \Gamma(\pi) \rightarrow \mathrm{E}_{p}$ is evaluation at $p$ and id $: \mathrm{V} \rightarrow \mathrm{V}$ is the identity operator. By linearity and continuity it follows that

for all $m \in M$.

$$
\widehat{m}(p)=\left(\mathrm{ev}_{p} \otimes \mathrm{id}\right)(m)
$$

Of course, $\widehat{m}(p)$ is actually $\Pi_{p}(m)$, where $\Pi_{p}: M \rightarrow \frac{M}{I_{p} M}=G_{p}$ is the natural surjection. Thus, we have a commuting diagram

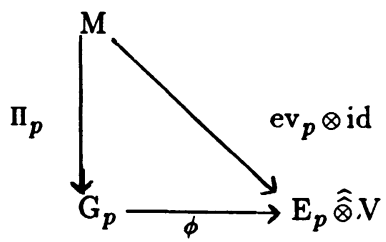

where $\phi$ is an isometric isomorphism. Thus, $\operatorname{ker}\left(\operatorname{ev}_{p} \otimes \mathrm{id}\right)=\operatorname{ker} \Pi_{p}=I_{p} M$, and since $\Pi_{p}$ is a quotient map, $\mathrm{ev}_{p} \otimes \mathrm{id}: \mathrm{M} \rightarrow \mathrm{E}_{p} \hat{\hat{\otimes}} \mathrm{V}$ is a quotient map also. $\mathrm{con}$

THEOREM 7. Let $\pi: \mathrm{E} \rightarrow \mathrm{X}$ and $\rho: \mathrm{F} \rightarrow \mathrm{X}$ be Banach bundles. Let $\mathrm{T}: \Gamma(\pi) \rightarrow \Gamma(\rho)$ be a bounded $\mathrm{C}(\mathrm{X})$-homomorphism, and for each $p \in \mathrm{X}$, let $\mathrm{T}_{p}: \mathrm{E}_{p} \rightarrow \mathrm{F}_{p}$ be the induced fiber map which results in the commutative diagram

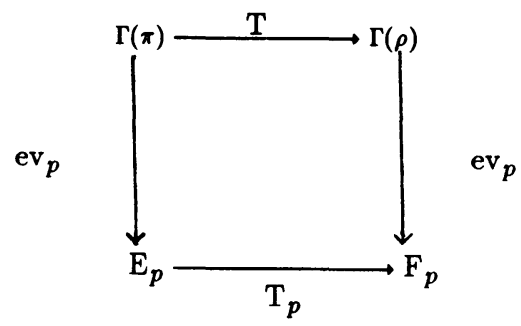

where $\mathrm{ev}_{p}$ denotes the evaluation of sections at $p$. If $\mathrm{T}: \Gamma(\pi) \rightarrow \Gamma(\rho)$ is an integral operator, then, for each $p \in \mathrm{X}, \mathrm{T}_{p}: \mathrm{E}_{p} \rightarrow \mathrm{F}_{p}$ is an integral operator. 
PROOF. Because $T$ is an integral operator, the map $\operatorname{ev}_{p} \circ T: \Gamma(\pi) \rightarrow F_{p}$ is also an integral operator. Thus, there is a unique bounded linear functional $\tau_{p}$ on $\Gamma(\pi) \hat{\hat{\otimes}}\left(F_{p}\right)^{*}$ such that

$$
\tau_{p}(\sigma \otimes f)=f\left(\left(\mathrm{ev}_{p} \circ \mathrm{T}\right)(\sigma)\right)=f(\{\mathrm{~T}(\sigma)\}(p))
$$

for all $\sigma \in \Gamma(\pi)$ and $f \in\left(\mathrm{F}_{p}\right)^{*}$.

According to Lemma $6, M=\Gamma(\pi) \hat{\hat{\otimes}}\left(F_{p}\right)^{*}$ can be made into a $C(X)$-module in such a way that

$$
a(\sigma \otimes f)=(a \sigma) \otimes f
$$

hold for all $a \in \mathrm{C}(\mathrm{X}), \sigma \in \Gamma(\pi)$, and $f \in\left(\mathrm{F}_{p}\right)^{*}$. Also, according to Lemma 6 , by taking the tensor product of $\mathrm{ev}_{p}: \Gamma(\pi) \rightarrow \mathrm{E}_{p}$ and the identity map id $:\left(\mathrm{F}_{p}\right)^{*} \rightarrow\left(\mathrm{F}_{p}\right)^{*}$ we get a quotient map $\operatorname{ev}_{p}: M \rightarrow E_{p} \hat{\hat{\otimes}}\left(F_{p}\right)^{*}$ whose kernel is $I_{p} M$.

Observe now that if $a \in \mathrm{C}(\mathrm{X}), \sigma \in \Gamma(\pi)$, and $f \in\left(\mathrm{F}_{p}\right)^{*}$, then

$$
\begin{aligned}
\tau_{p}(a(\sigma \otimes f))= & \tau_{p}((a \sigma) \otimes f)=f(\{\mathrm{~T}(a \sigma)\}(p)) \\
& =f(\{a \mathrm{~T}(\sigma)\})(p) \quad(\text { since } \mathrm{T} \text { is } \mathrm{C}(\mathrm{X}) \text {-linear }) \\
& =f(a(p)\{\mathrm{T}(\sigma)\}(p))=a(p) f(\{\mathrm{~T}(\sigma)\}(p))=a(p) \tau_{p}(\sigma \otimes f) .
\end{aligned}
$$

Because elements of the form $\sigma \otimes f$ span a dense subspace of $\Gamma(\pi) \hat{\hat{\otimes}}\left(\mathrm{F}_{p}\right)^{*}=\mathrm{M}$, and because $\tau_{p}$ is a bounded linear functional on $\mathrm{M}$, it follows that

$$
\tau_{p}(a m)=a(p) \tau_{p}(m)
$$

holds for all $a \in \mathrm{C}(\mathrm{X})$ and all $m \in \mathrm{M}$. It follows that the kernel of $\tau_{p}$ contains $\mathrm{I}_{p} \mathrm{M}$. Hence there is a bounded linear map $\phi: \frac{M}{I_{p} M} \rightarrow C$ such that $\tau_{p}=\phi \circ \tau_{p}=\phi \circ \Pi$, where $\Pi: M \rightarrow \frac{M}{I_{p} M}$ is the natural surjection. Since $\mathrm{ev}_{p} \otimes \mathrm{id}: \mathbf{M} \rightarrow \mathrm{E}_{p} \hat{\hat{\otimes}}\left(\mathrm{F}_{p}\right)^{*}$ is a quotient map whose kernel is $\mathrm{I}_{p} \mathrm{M}$, there is a surjective linear isometry $\Theta: \frac{M}{I_{p} M} \rightarrow E_{p} \hat{\otimes}\left(F_{p}\right)^{*}$ such that $e_{p} \otimes i d=\theta \circ I$. If we let $\widetilde{\phi}=\phi \circ \theta^{-1}$, then $\tilde{\phi}$ is a bounded linear functional on $E_{p} \hat{\hat{\otimes}}\left(F_{p}\right)^{*}$ and

$$
\tilde{\phi} \circ\left(\mathrm{ev}_{p} \otimes \mathrm{id}\right)=\left(\phi \circ \Theta^{-1}\right) \circ(\Theta \circ \Pi)=\phi \circ \Pi=\tau_{p} \text {. }
$$

Thus, we have the commutative diagram

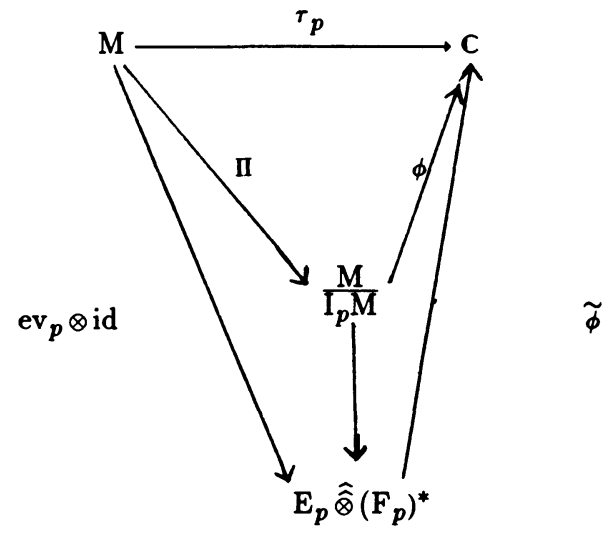

Now, let $e \in \mathrm{E}_{p}$ and $f \in\left(\mathrm{F}_{p}\right)^{*}$, and choose a section $\sigma \in \Gamma(\pi)$ such that $\sigma(p)=e$. Then

$$
\begin{aligned}
\tilde{\phi}(e \otimes f) & =\tilde{\phi}(\sigma(p) \otimes f)=\tilde{\phi}\left(\left(\mathrm{ev}_{p} \otimes \mathrm{id}\right)(\sigma \otimes f)\right) \\
& =\tau_{p}(\sigma \otimes f)=f\left(\left(\operatorname{ev}_{p} \circ \mathrm{T}\right)(\sigma)\right)=f\left(\left(\mathrm{~T}_{p} \circ \mathrm{ev}_{p}\right)(\sigma)\right) \\
& =f\left(\mathrm{~T}_{p}(\sigma(x))\right)=f\left(\mathrm{~T}_{p}(e)\right) .
\end{aligned}
$$

Since $\tilde{\phi}$ is bounded, this identity shows that $T_{p}$ is an integral operator. 000

Let $\mathrm{X}$ be a compact Hausdorff space, let $\mathrm{C} \subseteq \mathrm{X}$ be closed, and let $\mathrm{I}_{\mathrm{C}}=\{a \in \mathrm{C}(\mathrm{X}): a(\mathrm{C})=$ 0 . Let $\checkmark$ be the system of open neighborhoods of $C$. An approximate identity for $\mathrm{I}_{C}$ is a collection of continuous functions $\left\{i_{V}: \mathrm{X} \rightarrow[0,1]: V \in \mathbb{V}\right\}$ such that $i_{V}(\mathrm{C})=0$ and $i_{V}(\mathrm{X} \backslash V)=1$ for each $V \in \checkmark$. If $\Gamma(\pi)$ is the section space of the Banach bundle $\pi: \mathrm{E} \rightarrow \mathrm{X}$, then $\lim i_{V} \sigma=\sigma$ for 
each $\sigma \in \mathrm{I}_{C} \Gamma(\pi)$. It is known (see e.g. Gierz [10]) that for each closed $C \subseteq X, I_{C} \Gamma(\pi)$ is an $M$-ideal in $\Gamma(\pi)$, i.e. there is an $L^{1}$-projection $P_{C}: \Gamma(\pi)^{*} \rightarrow\left(I_{C} \Gamma(\pi)\right){ }^{\perp} \subseteq \Gamma(\pi)^{*}$. In particular, for $p \in X$, there is an $L^{1}$-projection $P_{p}: \Gamma(\pi)^{*} \rightarrow\left(I_{p} \Gamma(\pi)\right)^{\perp} \simeq\left(E_{p}\right)^{*} \subseteq \Gamma(\pi)^{*}$. We note that for $\phi \in \Gamma(\pi)^{*}$, $\phi \in\left(\mathrm{I}_{p} \Gamma(\pi)\right) \perp$ if and only if $a \phi=a(p) \phi$ for each $a \in \mathrm{C}(\mathrm{X})$. The next lemmas let us reach these $L^{1}$-projections $P_{p}$ as weak-* limits via approximate identities.

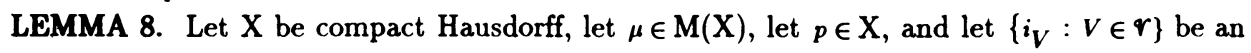
approximate identity for $I_{p}$. Then

$$
\lim \left\langle\left(1-i_{V}\right) \mu, a\right\rangle=\lim \int_{\mathrm{X}}\left(1-i_{V}\right) a \mathrm{~d} \mu=a(p) \mu(\{p\})
$$

for all $a \in \mathrm{C}(\mathrm{X})$. That is, the net $\left\{\left(1-i_{V}\right) \mu\right\}$ converges weak-* in $\mathrm{M}(\mathrm{X})$ to $\mu(\{p\}) \delta_{p}$, where $\delta_{p}$ is the unit point mass at $p$.

PROOF. We may assume that $\mu$ is a positive measure. Let $\epsilon>0$ be given. By the regularity of $\mu$, there is an open neighborhood $V_{0}$ of $p$ such that $\mu\left(V_{0}\right)<\mu(\{p\})+\epsilon$. Then for any open neighborhood $V$ of $p$ with $V \subseteq V_{0}$, we have

$$
\begin{aligned}
\mu(\{p\}) & =\int_{\{p\}}\left(1-i_{V}\right) \mathrm{d} \mu \leq \int_{V}\left(1-i_{V}\right) \mathrm{d} \mu \\
& \leq \int_{V} 1 \mathrm{~d} \mu=\mu(V) \leq \mu\left(V_{0}\right)<\mu(\{p\})+\epsilon .
\end{aligned}
$$

Thus, $\left|\int_{\mathrm{X}}\left(1-i_{V}\right) \mathrm{d} \mu-\mu(\{p\})\right|<\epsilon$, and $\lim \left\langle\left(1-i_{V}\right) \mu, 1\right\rangle=\mu(\{p\})$.

Let $a \in \mathrm{C}(\mathrm{X})$ be given, and consider now $b=a-a(p) \in \mathrm{I}_{p} \subseteq \mathrm{C}(\mathrm{X})$. Since $b \in \mathrm{I}_{p}$, we have $\left(1^{\prime}-i_{V}\right) b \rightarrow 0$ uniformly, so that $\left\langle\left(1-i_{V}\right) \mu, b\right\rangle=\int_{\mathrm{X}}\left(1-i_{V}\right) b \mathrm{~d} \mu \rightarrow 0$. Hence,

$$
\begin{aligned}
\lim \left\langle\left(1-i_{V}\right) \mu, a\right\rangle & =\lim \left\langle\left(1-i_{V}\right) \mu,(a-a(p)+a(p)\rangle\right. \\
& =\lim \left\langle\left(1-i_{V}\right) \mu, b\right\rangle+\lim \left\langle\left(1-i_{V}\right) \mu, a(p)\right\rangle \\
& =a(p) \mu(\{p\})=\left\langle\delta_{p}, a\right\rangle \mu(\{p\}) . \mathrm{DQD}
\end{aligned}
$$

LEMMA 9. Let $M=\Gamma(\pi)$, let $\phi \in M^{*}$, and suppose that $p \in X$. Let $\left\{i_{V}: V \in \mathcal{V}\right\}$ be an approximate identity for $I_{p}$. Then the net $\left\{\left(1-i_{V}\right) \phi\right\}$ converges weak-* to an element $\mathrm{P}_{p}(\phi) \in\left(\mathrm{I}_{p} \mathrm{M}\right) \stackrel{\perp}{\perp}\left(\mathrm{E}_{p}\right)^{*}$.

PROOF. Given $\phi \in \mathrm{M}^{*}$ and $\sigma \in \mathrm{M}$, we can define a functional $\phi^{\sigma} \in \mathrm{C}(\mathrm{X})^{*}=\mathrm{M}(\mathrm{X})$ by setting $\left\langle\phi^{\sigma}, a\right\rangle=\langle\phi, a \sigma\rangle$. We must show that $\lim \left\langle\left(1-i_{V}\right) \phi, \sigma\right\rangle$ exists. But for any $a \in \mathrm{C}(\mathrm{X})$, we have $\lim \left\langle\left(1-i_{V}\right) \phi, a \sigma\right\rangle=\lim \left\langle\phi,\left(1-i_{V}\right) a \sigma\right\rangle=\lim \left\langle\phi^{\sigma},\left(1-i_{V}\right) a\right\rangle=a(p) \phi^{\sigma}(\{p\})$, by the precerling lemma. Taking $a \equiv 1$, it follows that $\lim \left\langle\left(1-i_{V}\right) \phi, \sigma\right\rangle$ exists. Define this to be $\mathrm{P}_{p}(\phi) \in \Gamma(\pi)^{*}$; it clear that $\mathrm{P}_{p}$ is a bounded linear functional with $\left\|\mathrm{P}_{p}(\phi)\right\| \leq\|\phi\|$. Moreover,

$$
\left\langle a \mathrm{P}_{p}(\phi), \sigma\right\rangle=\left\langle\mathrm{P}_{p}(\phi), a \sigma\right\rangle=\lim \left\langle\left(1-i_{V}\right) \phi, a \sigma\right\rangle=a(p) \phi^{\sigma}(\{p\})=a(p)\left\langle\mathrm{P}_{p}(\phi), \sigma\right\rangle ;
$$

that is, $a \mathrm{P}_{p}(\phi)=a(p) \mathrm{P}_{p}(\phi)$ for all $a \in \mathrm{C}(\mathrm{X})$. Thus $\mathrm{P}_{p}(\phi) \in\left(\mathrm{I}_{p} \mathrm{M}\right) \stackrel{\perp}{\text {. }}$ वou

LEMMA 10. For $p \in X$, the map $P_{p}: \Gamma(\pi)^{*} \rightarrow \Gamma(\pi)^{*}$ is the unique $L^{1}$-projection of $\Gamma(\pi)^{*}$ onto $\left(\mathrm{I}_{p} \mathrm{M}\right)^{\perp}$.

PROOF. Clearly, $\mathrm{P}_{p}$ is a norm-decreasing linear map whose range is contained in $\left(\mathrm{I}_{p} \mathrm{M}\right)^{\perp}$. To show that it is a projection onto $\left(\mathrm{I}_{p} \mathrm{M}\right)^{\perp}$, it suffices to show that $\mathrm{P}_{p}(\phi)=\phi$ for all $\phi \in\left(\mathrm{I}_{p} \mathrm{M}\right)^{\perp}$. For such $\phi$, however, since $\left(1-i_{V}\right)(p)=1$ for all $V \in \mathbb{r}$.

$$
\mathrm{P}_{p}(\phi)=\lim \left(1-i_{V}\right) \phi=\lim \left(1-i_{V}\right)(p) \phi=\phi,
$$

To show that $\mathrm{P}_{p}$ is an $\mathrm{L}^{1}$-projection, it suffices to show that $\|\phi\| \geq\left\|\mathrm{P}_{p}(\phi)\right\|+\left\|\phi-\mathrm{P}_{p}(\phi)\right\|$ for each $\phi \in \Gamma(\pi)^{*}$. Let $\epsilon>0$ be given, and as before let $\left\{i_{V}: V \in \mathcal{Y}\right\}$ be an approximate identity for $I_{p}$. Choose sections $\sigma_{1}$ and $\sigma_{2}$ such that $\left\|\sigma_{1}\right\|=\left\|\sigma_{2}\right\|=1$ and such that

$$
\left.\left.\left\langle\mathrm{P}_{p}(\phi), \sigma_{1}\right\rangle+\epsilon\right\rangle\left\|\mathrm{P}_{p}\right\| \text { and }\left\langle\phi-\mathrm{P}_{p}(\phi), \sigma_{2}\right\rangle+\epsilon\right\rangle\left\|\phi-\mathrm{P}_{p}(\phi)\right\| \text {. }
$$

We may also choose $V_{0} \in \checkmark$ such that

$$
\left.\left\langle\phi, i_{V} \sigma_{2}\right\rangle+\epsilon=\left\langle\phi-\left(1-i_{V}\right) \phi, \sigma_{2}\right\rangle+\epsilon\right\rangle\left\|\phi-\mathrm{P}_{p}(\phi)\right\|
$$

for all $V \in \checkmark$ with $V \subseteq V_{0}$. For any such $V$, set $\tau=i_{V} \sigma_{2}+\left(1-i V^{2} \sigma_{1}\right.$. Then $\|\tau\| \leq 1$, and 


$$
\begin{aligned}
2 \epsilon+\langle\phi, \tau\rangle=2 \epsilon & +\left\langle\phi-\mathrm{P}_{p}(\phi), \tau\right\rangle+\left\langle\mathrm{P}_{p}(\phi), \tau\right\rangle \\
& =2 \epsilon+\left\langle\phi-\mathrm{P}_{p}(\phi), i_{V} \sigma_{2}+\left(1-i_{V}\right) \sigma_{1}\right\rangle+\left\langle\mathrm{P}_{p}(\phi), \sigma_{1}\right\rangle \\
& >\epsilon+\left\langle\phi, i_{V} \sigma_{2}\right\rangle-\left\langle\mathrm{P}_{p}(\phi), i_{V} \sigma_{2}\right\rangle+\left\langle\phi-\mathrm{P}_{p}(\phi),\left(1-i_{V}\right) \sigma_{1}\right\rangle+\left\|\mathrm{P}_{p}(\phi)\right\| \\
& >\left\|\phi-\mathrm{P}_{p}(\phi)\right\|+\left\|\mathrm{P}_{p}(\phi)\right\|+\left\langle\phi-\mathrm{P}_{p}(\phi),\left(1-i_{V}\right) \sigma_{1}\right\rangle
\end{aligned}
$$

(because $\left.\left\langle\mathrm{P}_{p}(\phi),{ }_{i} \sigma_{2}\right\rangle={ }^{2} V(p)\left\langle\mathrm{P}_{p}(\phi), \sigma_{2}\right\rangle=0\right)$. Thus,

$$
2 \epsilon+\|\phi\|>\left\|\phi-\mathrm{P}_{p}(\phi)\right\|+\left\|\mathrm{P}_{p}\right\|+\left\langle\phi-\mathrm{P}_{p}(\phi),\left(1-i_{V}\right) \sigma_{1}\right\rangle
$$

for all $V \in \mathbb{q}$ with $V \subseteq V_{0}$. Since

$$
\begin{aligned}
\lim \left\langle\phi-\mathrm{P}_{p}(\phi),\left(1-i_{V}\right) \sigma_{1}\right\rangle=\lim & \left\langle\left(1-i_{V}\right)\left(\phi-\mathrm{P}_{p}(\phi), \sigma_{1}\right\rangle\right. \\
& =\left\langle\mathrm{P}_{p}\left(\phi-\mathrm{P}_{p}(\phi)\right), \sigma_{1}\right\rangle=\left\langle\mathrm{P}_{p}(\phi)-\mathrm{P}_{p}(\phi), \sigma_{1}\right\rangle=0,
\end{aligned}
$$

it follows that

$$
2 \epsilon+\|\phi\|>\left\|\mathrm{P}_{p}(\phi)\right\|+\left\|\phi-\mathrm{P}_{p}(\phi)\right\| \cdot \mathrm{QD0}
$$

(Actually, more is true than this string of lemmas would indicate. It can be shown that we actually have $\left(1-i_{V}\right) \phi \rightarrow \mathrm{P}_{\mathrm{C}}(\phi)$ in the norm topology of $\Gamma(\pi)^{*}$ whenever $\mathrm{C} \subseteq \mathrm{X}$ is closed and $\left\{i_{V}\right\}$ is an approximate identity for $\mathrm{I}_{\mathrm{C}} \subseteq \mathrm{C}(\mathrm{X})$. However, our proof uses the preceding lemmas, and we do not need this more general result in what follows.)

We may regard the space $M=\operatorname{Int}_{X}(\Gamma(\pi), \Gamma(\rho))$ of integral operators from $\Gamma(\pi)$ to $\Gamma(\rho)$ which are $\mathrm{C}(\mathrm{X})$-module homomorphisms as a Banach space under the integral norm (Diestel and Uhl [7]) ; that $\mathrm{M}$ is a $\mathrm{C}(\mathrm{X})$-module follows because, for $\mathrm{T} \in \mathrm{M}, a \in \mathrm{C}(\mathrm{X})$, and $\sigma \in \Gamma(\pi)$ we have $(a \mathrm{~T})(\sigma)$ $=a \bullet T(\sigma)=\left(\mu_{a} \circ \mathrm{T}\right)(\sigma)$, where $\mu_{a}$ is the operator $\tau \mapsto a \tau$ on $\Gamma(\rho)$. Our final result involves the fibers of the canonical bundle of $\mathrm{M}$ as a $\mathrm{C}(\mathrm{X})$-module.

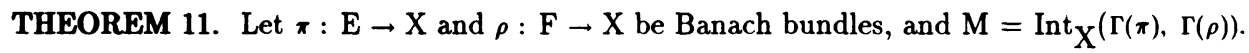
If $\mathrm{T} \in \mathrm{M}$, then $\left\|\mathrm{T}+\mathrm{I}_{p} \mathrm{M}\right\|_{\text {int }}=\left\|\mathrm{T}_{p}\right\|_{\text {int }}=\inf \quad\left\{\left\|\mathrm{T}+a \mathrm{~T}^{\prime}\right\|\right.$ int $\left.: a \in \mathrm{I}_{p}, \mathrm{~T}^{\prime} \in \mathrm{M}\right\}$, where $\mathrm{T}_{p}: \mathrm{E}_{p} \rightarrow \mathrm{F}_{p}$ is the induced map described in the statement of Theorem 7. Thus, the map $\mathrm{T} \mapsto$ $\mathrm{T}_{p}$ is a quotient map, and if $\xi: \mathrm{G} \rightarrow \mathrm{X}$ is the canonical bundle for $\mathrm{M}$, then for each $p \in \mathrm{X}$ there is a isometry $\alpha_{p}$ from $G_{p}$, the fiber over $p$, to $\operatorname{Int}\left(\mathrm{E}_{p}, \mathrm{~F}_{p}\right)$, the space of integral maps from $\mathrm{E}_{p}$ to $\mathrm{F}_{p}$, with $\alpha_{p}\left(\mathrm{~T}+\mathrm{I}_{p} \mathrm{M}\right)=\mathrm{T}_{p}$.

PROOF. In this proof, all norms of operators in $M$ are assumed to be the integral norms, and norms of cosets are arrived at by taking infima of integral norms.

Let $\mathrm{T}, \mathrm{T}^{\prime} \in \mathrm{M}, a \in \mathrm{I}_{p}$, and let $\epsilon>0$ be given. We may choose an element $\sum \sigma_{k} \otimes y_{k}^{*} \in \Gamma(x) \hat{\otimes} \Gamma(\rho)^{*}$ such that $\left\|\sum \sigma_{k} \otimes y_{k}^{*}\right\|^{\hat{}}=1$ and such that

$$
\left\|\mathrm{T}+a \mathrm{~T}^{\prime}\right\|<\left|\sum\left\langle y_{k}^{*},\left(\mathrm{~T}+a \mathrm{~T}^{\prime}\right)\left(\sigma_{k}\right)\right\rangle\right|+\epsilon .
$$

Let $\left\{{ }^{i}\right\}$ be an approximate identity for $\mathrm{I}_{p}$. For any $V \in \boldsymbol{q}$, we have

$$
\begin{aligned}
\| \mathrm{T}+a \mathrm{~T}^{\prime} & \|<\left|\sum\left\langle y_{k}^{*},\left(1-i_{V}\right)\left(\mathrm{T}+a \mathrm{~T}^{\prime}\right)\left(\sigma_{k}\right)\right\rangle\right|+\left|\sum\left\langle y_{k}^{*}, i_{V}\left(\mathrm{~T}+a \mathrm{~T}^{\prime}\right)\left(\sigma_{k}\right)\right\rangle\right|+\epsilon \\
& \leq\left|\sum\left\langle y_{k}^{*},\left(1-i_{V}\right)\left(\mathrm{T}+a \mathrm{~T}^{\prime}\right)\left(\sigma_{k}\right)\right\rangle\right|+\left\|i_{V}\left(\mathrm{~T}+a \mathrm{~T}^{\prime}\right)\right\|+\epsilon \\
& \leq\left|\sum\left\langle y_{k}^{*},\left(1-i_{V}\right) \mathrm{T}\left(\sigma_{k}\right)\right\rangle\right|+\left|\sum\left\langle y_{k}^{*},\left(1-i_{V}\right)\left(a \mathrm{~T}^{\prime}\right)\left(\sigma_{k}\right)\right\rangle\right|+\left\|{ }^{i} V\left(\mathrm{~T}+a \mathrm{~T}^{\prime}\right)\right\|+\epsilon .
\end{aligned}
$$

Hence,

$$
\begin{aligned}
\left\|\mathrm{T}+\mathrm{I}_{p} \mathrm{M}\right\|= & \inf \left\{\left\|\mathrm{T}+a \mathrm{~T}^{\prime}\right\|: a \in \mathrm{I}_{p}, \mathrm{~T}^{\prime} \in \mathrm{M}\right\} \\
& \leq \inf _{a \mathrm{~T}^{\prime}} \inf _{V}\left\{\left|\sum\left\langle y_{k}^{*},\left(1-i_{V}\right) \mathrm{T}\left(\sigma_{k}\right)\right\rangle\right|+\left|\sum\left\langle y_{k}^{*},\left(1-i_{V}\right)\left(a \mathrm{~T}^{\prime}\right)\left(\sigma_{k}\right)\right\rangle\right|\right. \\
& \left.+\left\|i_{V}\left(\mathrm{~T}+a \mathrm{~T}^{\prime}\right)\right\|+\epsilon\right\},
\end{aligned}
$$

where of course the choice of $\sum \sigma_{k} \otimes y_{k}^{*} \in \Gamma(\pi) \hat{\hat{\otimes}} \Gamma(\rho)^{*}$ depends on our choice of $a \mathrm{~T}^{\prime}$.

Now, denote by $\mathrm{P}_{p}: \Gamma(\rho)^{*} \rightarrow\left(\mathrm{F}_{p}\right)^{*}$ the $\mathrm{L}^{1}$-projection; from Lemma 10 , for each $y^{*} \in \Gamma(\rho)^{*}$ we have $\left(1-i_{V}\right) y^{*} \rightarrow \mathrm{P}_{p}\left(y^{*}\right)$ pointwise on $\Gamma(\rho)$. For a fixed but arbitrary $a \mathrm{~T}^{\prime} \in \mathrm{I}_{p} \mathrm{M}$, we may choose 
$V_{0}=V_{0}\left(a T^{\prime}\right)$ such that

and such that

$$
\left|\sum\left\langle y_{k}^{*},\left(1-i V_{0}\right) \mathrm{T}\left(\sigma_{k}\right)\right\rangle\right|<\left|\sum\left\langle\mathrm{P}_{p}\left(y_{k}^{*}\right), \mathrm{T}_{p}\left(\sigma_{k}(p)\right)\right\rangle\right|+\epsilon
$$

since $\left\{\left(a \mathrm{~T}^{\prime}\right)\left(\sigma_{k}\right)\right\}(p)=a(p)\left\{\mathrm{T}^{\prime}\left(\sigma_{k}\right)\right\}(p)=0$.

$$
\left|\sum\left\langle y_{k}^{*},\left(1-i_{V_{0}}\right)\left(a \mathrm{~T}^{\prime}\right)\left(\sigma_{k}\right)\right\rangle\right|<\left|\sum\left\langle\mathrm{P}_{p}\left(y_{k}^{*}\right),\left(a \mathrm{~T}^{\prime}\right)\left(\sigma_{k}(p)\right)\right\rangle\right|+\epsilon=\epsilon
$$

Hence,

$$
\begin{aligned}
\| \mathrm{T}+ & \mathrm{I}_{p} \mathrm{M} \| \\
& \leq \inf _{a \mathrm{~T}^{\prime}} \inf _{V}\left\{\left|\sum\left\langle y_{k}^{*},\left(1-i_{V}\right) \mathrm{T}\left(\sigma_{k}\right)\right\rangle\right|+\left|\sum y_{k}^{*},\left(1-i_{V}\right)\left(a \mathrm{~T}^{\prime}\right)\left(\sigma_{k}\right)\right\rangle \mid+\left\|i_{V}\left(\mathrm{~T}+a \mathrm{~T}^{\prime}\right)\right\|+\epsilon\right\} \\
& \leq \inf _{a \mathrm{~T}^{\prime}} \inf _{V}\left\{\left|\sum\left\langle\mathrm{P}_{p}\left(y_{k}^{*}\right), \mathrm{T}_{p}\left(\sigma_{k}(p)\right)\right\rangle\right|+\epsilon+\epsilon+\left\|i_{V}\left(\mathrm{~T}+a \mathrm{~T}^{\prime}\right)\right\|+\epsilon\right\} \\
& =\inf _{a \mathrm{~T}^{\prime}} \inf _{V}\left\{\left|\sum\left\langle\mathrm{P}_{p}\left(y_{k}^{*}\right), \mathrm{T}_{p}\left(\sigma_{k}(p)\right)\right\rangle\right|+\left\|i_{V}\left(\mathrm{~T}+a \mathrm{~T}^{\prime}\right)\right\|+3 \epsilon\right\} .
\end{aligned}
$$

But $\left|\sum\left\langle\mathrm{P}_{p}\left(y_{k}^{*}\right), \mathrm{T}_{p}\left(\sigma_{k}(p)\right)\right\rangle\right| \leq\left\|\mathrm{T}_{p}\right\|$, since the map $\operatorname{ev}_{p} \otimes \mathrm{P}_{p}: \Gamma(\pi) \hat{\hat{\otimes}} \Gamma(\rho)^{*} \rightarrow \mathrm{E}_{p} \hat{\hat{\otimes}}\left(\mathrm{F}_{p}\right)^{*}$, $\sigma \otimes y^{*} \mapsto \sigma(p) \otimes \mathrm{P}_{p}\left(y^{*}\right)$ has norm $\leq 1$. Thus, .

$$
\begin{aligned}
\left\|\mathrm{T}+\mathrm{I}_{p} \mathrm{M}\right\| & \leq \inf _{a \mathrm{~T}^{\prime}} \inf _{V}\left\{\left|\sum\left\langle\mathrm{P}_{p}\left(y_{k}^{*}\right), \mathrm{T}_{p}\left(\sigma_{k}(p)\right)\right\rangle\right|+\left\|i_{V}\left(\mathrm{~T}+a \mathrm{~T}^{\prime}\right)\right\|+3 \epsilon\right\} \\
& \leq \inf _{a \mathrm{~T}^{\prime}} \inf _{V}\left\{\left\|\mathrm{~T}_{p}\right\|+\left\|{ }^{i} V\left(\mathrm{~T}+a \mathrm{~T}^{\prime}\right)\right\|+3 \epsilon\right\} \\
& =\inf _{V} \inf _{a \mathrm{~T}^{\prime}}\left\{\left\|\mathrm{T}_{p}\right\|+\left\|{ }^{i} V\left(\mathrm{~T}+a \mathrm{~T}^{\prime}\right)\right\|+3 \epsilon\right\} \\
& =\inf _{V}\left\{\left\|\mathrm{~T}_{p}\right\|+\left\|\mathrm{i}_{V} \mathrm{~T}+\mathrm{I}_{p} \mathrm{M}\right\|+3 \epsilon\right\} \\
& =\left\|\mathrm{T}_{p}\right\|+3 \epsilon .
\end{aligned}
$$

Since $\epsilon$ was arbitrary, we have $\left\|\mathrm{T}+\mathrm{I}_{p} \mathrm{M}\right\| \leq\left\|\mathrm{T}_{p}\right\|$. $\mathrm{COD}$

In much the same fashion as above, it may be shown that if $\mathrm{V}$ is a Banach space and $\pi: \mathrm{E}$ $\rightarrow X$ is a Banach bundle, then the space $M=\operatorname{Int}(V, \Gamma(\pi))$ of integral operators from $V$ to $\Gamma(\pi)$ is a $\mathrm{C}(\mathrm{X})$-module. The fiber over $p$ of the canonical bundle for $\mathrm{M}$ then turns out to be $\mathrm{ev}_{1}, \circ \mathrm{T}$. This is analogous to the result in Gierz [11], regarding compact maps from $V$ to $\Gamma(\pi)$.

ACKNOWLEDGMENT. The authors wish to thank the referee for several valuable sugges r ons, including an improvement in the proof of Theorem 3.

\section{REFERENCES}

1. SAAB, P., Integral operators on spaces of continuous vector-valued functions, Proc. Anner. Math. Soc. 111 (1991), 1003-1013.

2. GROTHENDIECK, A., Produits tensoriels topologiques et espaces nucléaires, Mem. Amer. Math. Soc. 16 (1955).

3. KITCHEN, J.W. \& ROBBINS, D.A., Gelfand representation of banach modules, Dissertations Math. (Rozprawy Mat.) $203(1982 / 1983)$.

4. SEDA, A., On the categories $S p(X)$ and $B a n(X)$, Cahiers Topo. et Geom. Diff. XXIV (1983), 97-112.

5. KITCHEN, J.W. \& ROBBINS, D.A., Internal functionals and bundle duals, Internat. J. Math. \& Math. Sci. 7 (1984), 689-695.

6. GIERZ, G., Bundles of topological vector spaces and their duality, Lect. Notes in Math. 955 Springer Verlag, 1982.

7. DIESTEL, J \& UHL, J., Vector measures, Amer. Math. Soc. Surveys 15 (1977). 8. BEHRENDS, E., M-structure and the Banach-Stone theorem, Lect. Notes in Math. 736,
Springer-Verlag, 1979 .

9. KITCHEN, J.W. \& ROBBINS, D.A., Tensor products of Banach bundles, Pac. J. Math. 94 (1981), 151-169.

10. GIERZ, G., Integral representations of linear functional on spaces of sections in separable bundles, Rocky Mt. J. Math. 17 (1987), 545-554.

11. GIERZ, G., Representation of spaces of compact operators and applications to the approximation property, Arch. Math. (Basel) 30 (1978), 622-628. 


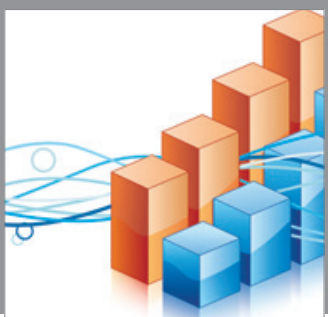

Advances in

Operations Research

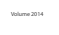

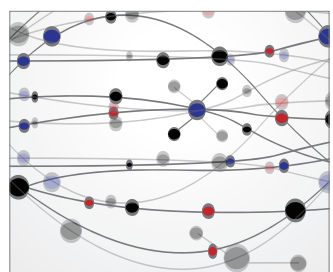

\section{The Scientific} World Journal
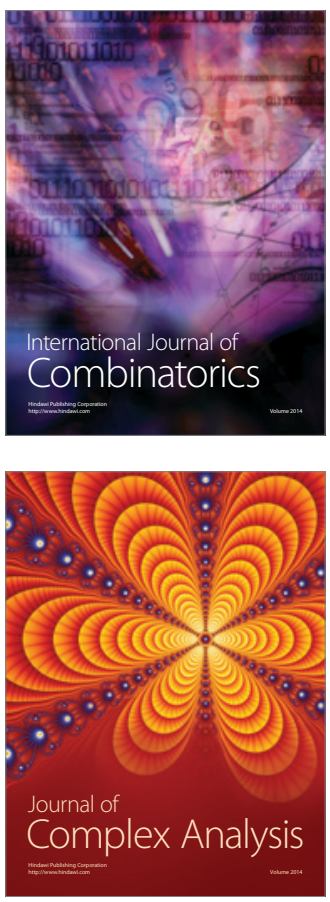

International Journal of

Mathematics and

Mathematical

Sciences
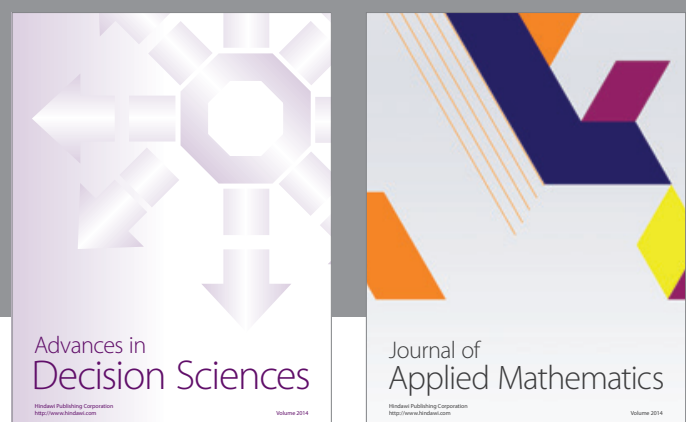

Journal of

Applied Mathematics
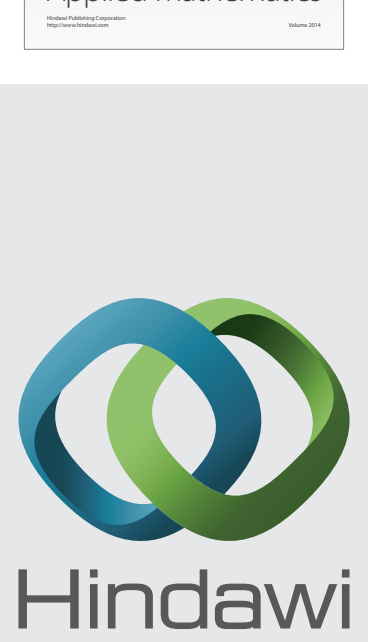

Submit your manuscripts at http://www.hindawi.com
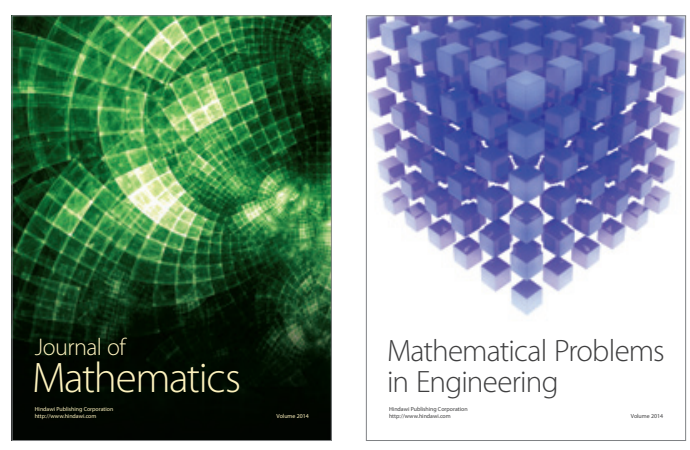

Mathematical Problems in Engineering
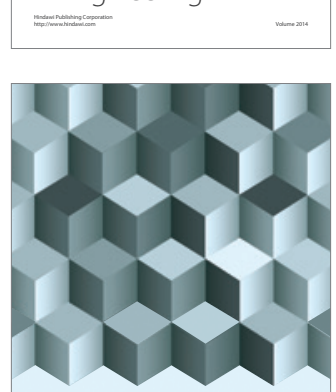

Journal of

Function Spaces
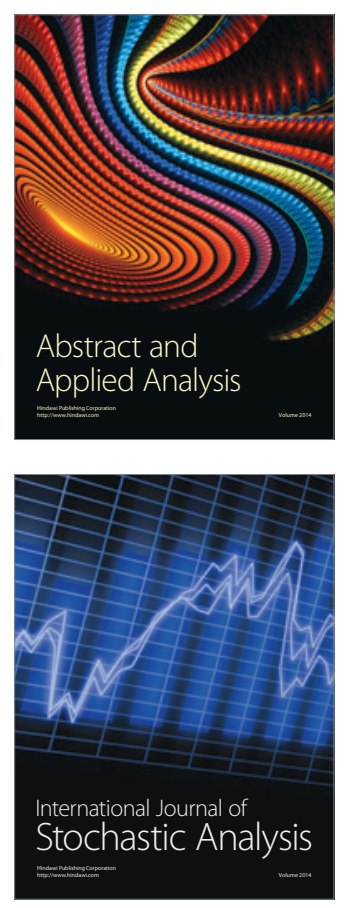

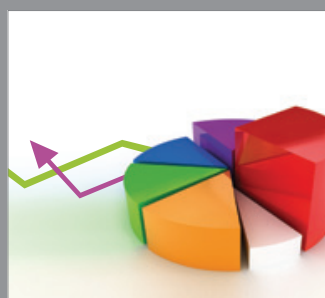

ournal of

Probability and Statistics

Promensencen
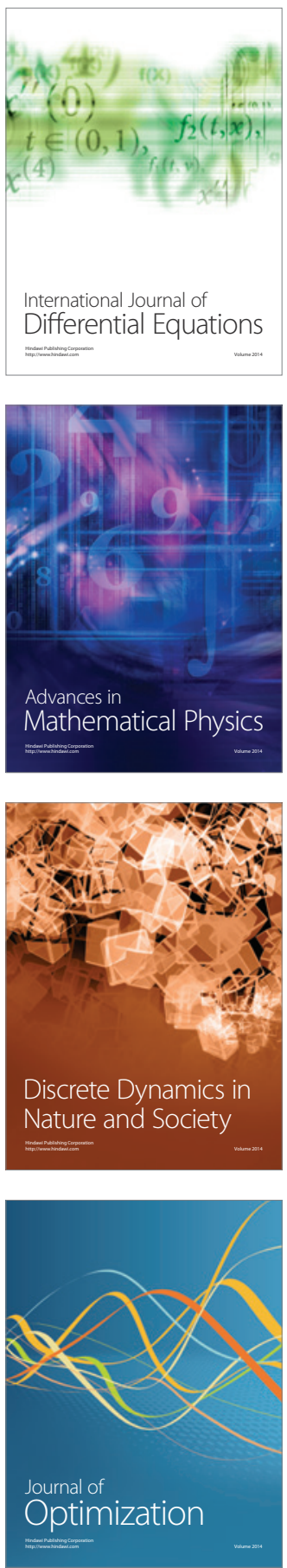